\title{
A GENERALIZATION OF THE WIDDER-ARENDT THEOREM
}

\author{
WOJCIECH CHOJNACKI \\ Wydziat Matematyki, Uniwersytet Kardynata Stefana Wyszyńskiego, \\ ul. Dewajtis 5, 01-815 Warszawa, Poland, and Department of Computer Science, \\ University of Adelaide, Adelaide, SA 5005, Australia \\ (chojnacki@uksw.edu.pl; wojtek@cs.adelaide.edu.au)
}

(Received 27 July 1999)

\begin{abstract}
We establish a generalization of the Widder-Arendt theorem from Laplace transform theory. Given a Banach space $E$, a non-negative Borel measure $m$ on the set $\mathbb{R}_{+}$of all non-negative numbers, and an element $\omega$ of $\mathbb{R} \cup\{-\infty\}$ such that $\epsilon_{-\lambda}$ is m-integrable for all $\lambda>\omega$, where $\epsilon_{-\lambda}$ is defined by $\epsilon_{-\lambda}(t)=$ $\exp (-\lambda t)$ for all $t \in \mathbb{R}_{+}$, our generalization gives an intrinsic description of functions $r:(\omega, \infty) \rightarrow E$ that can be represented as $r(\lambda)=T\left(\epsilon_{-\lambda}\right)$ for some bounded linear operator $T: L^{1}\left(\mathbb{R}_{+}, \mathrm{m}\right) \rightarrow E$ and all $\lambda>\omega$; here $L^{1}\left(\mathbb{R}_{+}, \mathrm{m}\right)$ denotes the Lebesgue space based on $\mathrm{m}$. We use this result to characterize pseudo-resolvents with values in a Banach algebra, satisfying a growth condition of Hille-Yosida type.
\end{abstract}

Keywords: Laplace-Stieltjes transform; weighted convolution algebra; representation; pseudo-resolvent; one-parameter semigroup

AMS 2000 Mathematics subject classification: Primary 44A10; 47A10

Secondary 43A20; 46B22; 46G10; 46J25; 47D06

\section{Introduction}

Let $\mathbb{F}$ be either the field $\mathbb{R}$ of real numbers or the field $\mathbb{C}$ of complex numbers. Hereafter all vector spaces will be assumed to be over $\mathbb{F}$. Any particular choice of the ground field $\mathbb{F}$ will be inessential for the validity of results. Let $\mathbb{R}_{+}$be the set of all non-negative numbers and let $\mathbb{R}_{+}^{\bullet}$ be the set of all positive numbers. A weight function on $\mathbb{R}_{+}$is a Lebesgue measurable function $\Omega: \mathbb{R}_{+} \rightarrow \mathbb{R}_{+}^{\bullet}$ such that

$$
\Omega(s+t) \leqslant \Omega(s) \Omega(t)
$$

for all $s, t \in \mathbb{R}_{+}$. A function satisfying (1.1) is said to be submultiplicative. Given a weight function $\Omega$, denote by $L^{1}\left(\mathbb{R}_{+}, \Omega\right)$ the set of equivalence classes of $\mathbb{F}$-valued Lebesgue measurable functions $f$ on $\mathbb{R}_{+}$such that

$$
\|f\|_{1, \Omega}=\int_{\mathbb{R}_{+}}|f(t)| \Omega(t) \mathrm{d} t<\infty,
$$

where two functions are equivalent if they are equal almost everywhere. With addition and scalar multiplication derived from the addition and scalar multiplication of functions, 
and with the norm $\|\cdot\|_{1, \Omega}, L^{1}\left(\mathbb{R}_{+}, \Omega\right)$ is a Banach space. Moreover, $L^{1}\left(\mathbb{R}_{+}, \Omega\right)$ is a Banach algebra, a weighted convolution algebra, when multiplication is defined to be the convolution

$$
\left.(f * g)(t)=\int_{0}^{t} f(t-s) g(s) \mathrm{d} s \quad \text { (almost all } t \in \mathbb{R}_{+}\right) .
$$

Let $\mathbb{Z}^{+}$be the set of all non-negative integers. For each $\lambda \in \mathbb{R}$, denote by $\epsilon_{\lambda}$ the function

$$
\epsilon_{\lambda}(t)=\mathrm{e}^{\lambda t} \quad\left(t \in \mathbb{R}_{+}\right)
$$

We shall employ the notation

$$
f^{* n}=\underbrace{f * \cdots * f}_{n \text { times }} .
$$

For each $k \in \mathbb{Z}^{+}$and each $\lambda \in \mathbb{R}$, set

$$
\begin{aligned}
\alpha_{k, \lambda} & =\epsilon_{-\lambda}^{*(k+1)} \\
\beta_{k, \lambda} & =k ! \alpha_{k, \lambda} \\
\gamma_{k, \lambda} & =\lambda^{k} \alpha_{k, \lambda}
\end{aligned}
$$

more explicitly,

$$
\alpha_{k, \lambda}(t)=\frac{t^{k}}{k !} \mathrm{e}^{-\lambda t}, \quad \beta_{k, \lambda}(t)=t^{k} \mathrm{e}^{-\lambda t}, \quad \gamma_{k, \lambda}(t)=\frac{(\lambda t)^{k}}{k !} \mathrm{e}^{-\lambda t}
$$

for all $t \in \mathbb{R}_{+}$.

Let $\Omega$ be a weight function on $\mathbb{R}_{+}$. A bound for $\Omega$ is an element $\omega$ of $\{-\infty\} \cup \mathbb{R}$ such that $\epsilon_{-\mu} \in L^{1}\left(\mathbb{R}_{+}, \Omega\right)$ for each $\mu>\omega$. If $\omega$ is a bound for $\Omega$, then $\alpha_{k, \mu} \in L^{1}\left(\mathbb{R}_{+}, \Omega\right.$ ) (and automatically $\beta_{k, \mu} \in L^{1}\left(\mathbb{R}_{+}, \Omega\right)$ and $\left.\gamma_{k, \mu} \in L^{1}\left(\mathbb{R}_{+}, \Omega\right)\right)$ for all $k \in \mathbb{Z}^{+}$and all $\mu>\omega$.

Let $E$ be a Banach space. Given an open subset $U$ of $\mathbb{R}$, let $C^{\infty}(U, E)$ be the space of $E$-valued functions on $U$ infinitely differentiable in the norm topology of $E$. Let $\Omega$ be a weight function on $\mathbb{R}_{+}$with bound $\omega$. For $r \in C^{\infty}((\omega, \infty), E)$, set

$$
\|r\|_{W, \Omega, \omega}=\sup \left\{\left\|r^{(k)}(\lambda)\right\| /\left\|\beta_{k, \lambda}\right\|_{1, \Omega} \mid k \in \mathbb{Z}^{+}, \lambda \in(\omega, \infty)\right\}
$$

and define the Widder space $C_{W}^{\infty}((\omega, \infty), E ; \Omega)$ by

$$
C_{W}^{\infty}((\omega, \infty), E ; \Omega)=\left\{r \in C^{\infty}((\omega, \infty), E)\|\| r \|_{W, \Omega, \omega}<\infty\right\}
$$

Equipped with the norm $\|\cdot\|_{W, \Omega, \omega}, C_{W}^{\infty}((\omega, \infty), E ; \Omega)$ is a Banach space.

A weight function $\Omega: \mathbb{R}_{+} \rightarrow \mathbb{R}_{+}^{\bullet}$ will be termed an $(\alpha, \omega)$-weight function if

(i) $\Omega$ is continuous and satisfies $\Omega(0)=1$;

(ii) there exist $\alpha \in \mathbb{R}_{+}$and $\omega \in \mathbb{R}$ such that $\Omega(t) t^{-\alpha} \mathrm{e}^{-\omega t}$ tends to a positive number as $t \rightarrow \infty$. 
The numbers $\alpha$ and $\omega$ in condition (ii) are uniquely determined and define the power order and exponential order of $\Omega$, respectively. A simple example of an $(\alpha, \omega)$-weight function is furnished by

$$
\Omega(t)=(1+\beta t)^{\alpha} \mathrm{e}^{\omega t} \quad\left(t \in \mathbb{R}_{+}\right)
$$

with $\alpha, \beta \in \mathbb{R}_{+}$and $\omega \in \mathbb{R}$. It is readily verified that if $\Omega$ is an $(\alpha, \omega)$-weight function, then $\omega$ is a bound for $L^{1}\left(\mathbb{R}_{+}, \Omega\right)$.

Given two normed vector spaces $E$ and $F$, denote by $\mathcal{L}(E, F)$ the space of all bounded linear operators from $E$ into $F$.

Recently, Kisyński [17] established the following result.

Theorem 1.1 (Widder-Arendt-Kisyński). Let $\Omega$ be an $(\alpha, \omega)$-weight function on $\mathbb{R}_{+}$, let $E$ be a Banach space, and let $r:(\omega, \infty) \rightarrow E$ be a function. Then $r$ can be represented in the form

$$
r(\lambda)=T\left(\epsilon_{-\lambda}\right) \quad(\lambda>\omega)
$$

for some $T \in \mathcal{L}\left(L^{1}\left(\mathbb{R}_{+}, \Omega\right), E\right)$ if and only if $r \in C_{W}^{\infty}((\omega, \infty), E ; \Omega)$. If there exists a $T \in \mathcal{L}\left(L^{1}\left(\mathbb{R}_{+}, \Omega\right), E\right)$ such that $r(\lambda)=T\left(\epsilon_{-\lambda}\right)$ for all $\lambda>\omega$, then $T$ is unique and $\|T\|=\|r\|_{W, \Omega, \omega}$.

This theorem is a generalization of the Widder-Arendt representation theorem from Laplace transform theory. Widder established Theorem 1.1 in the case that $E=\mathbb{F}$ and $\Omega$ is of the form $\Omega(t)=\mathrm{e}^{\omega t}\left(t \in \mathbb{R}_{+}, \omega \in \mathbb{R}\right.$ ) (cf. [21, pp. 315-316] and [22, p. 157]). Since $\mathcal{L}\left(L^{1}\left(\mathbb{R}_{+}, \Omega\right), \mathbb{F}\right)$ is isometrically isomorphic to the space $L^{\infty}\left(\mathbb{R}_{+}, \Omega^{-1}\right)$ of all (equivalence classes of) $\mathbb{F}$-valued Lebesgue measurable functions $f$ on $\mathbb{R}_{+}$for which $f \Omega^{-1}$ is essentially bounded, Theorem 1.1 in that case can be viewed as a characterization of the Laplace transforms of elements of $L^{\infty}\left(\mathbb{R}_{+}, \Omega^{-1}\right)$. Extension to the case in which $E$ is not necessarily $\mathbb{F}$ and $\Omega$ is still of the form $\Omega(t)=\mathrm{e}^{\omega t}\left(t \in \mathbb{R}_{+}, \omega \in \mathbb{R}\right)$ is due to Arendt [1]. Arendt's approach, drawing on Widder's result, relies on reduction of the vector case to the scalar one. A direct proof of Arendt's result was given by Hennig and Neubrander [14] (see also [18]). Another proof was offered by Bobrowski [3]. deLaubenfels et al. [7] proved a special case of Theorem 1.1 in which $\Omega(t)=(1+t)^{k}\left(t \in \mathbb{R}_{+}, k \in \mathbb{Z}^{+}\right)$. Kisyński established a simultaneous generalization of the results of Arendt and deLaubenfels et al..

The aim of this paper is establish a generalization of Theorem 1.1. In this generalization, formulated as Theorem 1.2 below, $L^{1}\left(\mathbb{R}_{+}, \Omega\right)$ is replaced by a more general $L^{1}$ space, not necessarily a weighted convolution algebra. This space may in particular be of the form $L^{1}\left(\mathbb{R}_{+}, \Omega\right)$, where $\Omega$ is a weight function with a bound, e.g. an $(\alpha, \omega)$-weight function. A similar result was recently, independently obtained by Bobrowski [4].

Let $\mathrm{m}$ be a non-negative Borel measure on $\mathbb{R}_{+}$. Let $L^{1}\left(\mathbb{R}_{+}, \mathrm{m}\right.$ ) be the space of all (classes of) $\mathbb{F}$-valued $m$-integrable functions on $\mathbb{R}_{+}$. A bound for $m$ is an element $\omega$ of $\{-\infty\} \cup \mathbb{R}$ such that $\epsilon_{-\mu} \in L^{1}\left(\mathbb{R}_{+}, \mathrm{m}\right)$ for each $\mu>\omega$. If $\omega$ is a bound for $\mathrm{m}$, then $\alpha_{k, \mu} \in L^{1}\left(\mathbb{R}_{+}, \mathrm{m}\right)$ (and hence also $\beta_{k, \mu} \in L^{1}\left(\mathbb{R}_{+}, \mathrm{m}\right)$ and $\gamma_{k, \mu} \in L^{1}\left(\mathbb{R}_{+}, \mathrm{m}\right)$ ) for all $k \in \mathbb{Z}^{+}$and all $\mu>\omega$. Note that if a measure $\mathrm{m}$ admits a bound, then it is Radon; that is to say, $\mathrm{m}$ is finite on every bounded Borel subset of $\mathbb{R}_{+}$. 
Let $\mathrm{m}$ be a non-negative Borel measure on $\mathbb{R}_{+}$with bound $\omega$. Let $E$ be a Banach space. For $r \in C^{\infty}((\omega, \infty), E)$, set

$$
\|r\|_{W, \mathrm{~m}, \omega}=\sup \left\{\left\|r^{(k)}(\lambda)\right\| /\left\|\beta_{k, \lambda}\right\|_{1, \mathrm{~m}} \mid k \in \mathbb{Z}^{+}, \lambda \in(\omega, \infty)\right\}
$$

and define the Widder space $C_{W}^{\infty}((\omega, \infty), E ; \mathrm{m})$ by

$$
C_{W}^{\infty}((\omega, \infty), E ; \mathrm{m})=\left\{r \in C^{\infty}((\omega, \infty), E) \mid\|r\|_{W, \mathrm{~m}, \omega}<\infty\right\} .
$$

Endowed with the norm $\|\cdot\|_{W, \mathrm{~m}, \omega}, C_{W}^{\infty}((\omega, \infty), E ; \mathrm{m})$ is a Banach space.

The main result of this paper is the following theorem.

Theorem 1.2. Let $\mathrm{m}$ be a non-negative Borel measure on $\mathbb{R}_{+}$with bound $\omega$, let $E$ be a Banach space, and let $r:(\omega, \infty) \rightarrow E$ be a function. Then $r$ can be represented in the form

$$
r(\lambda)=T\left(\epsilon_{-\lambda}\right) \quad(\lambda>\omega)
$$

for some $T \in \mathcal{L}\left(L^{1}\left(\mathbb{R}_{+}, \mathrm{m}\right), E\right)$ if and only if $r \in C_{W}^{\infty}((\omega, \infty), E ; \mathrm{m})$. If there exists a $T \in \mathcal{L}\left(L^{1}\left(\mathbb{R}_{+}, \mathrm{m}\right), E\right)$ such that $r(\lambda)=T\left(\epsilon_{-\lambda}\right)$ for all $\lambda>\omega$, then $T$ is unique and $\|T\|=\|r\|_{W, \mathrm{~m}, \omega}$.

As is the case with all versions of the Widder-Arendt theorem, the proof of Theorem 1.2 is based on an approximation argument. The method we use draws on the technique applied by Kisyński in [16] to prove the classical Widder-Arendt theorem. Bobrowski proved a result similar to Theorem 1.2 employing the same technique with which he had earlier re-established the classical Widder-Arendt theorem (cf. [2]) and which goes back to Phillips [19] (see also [15, Theorem 6.6.3]).

The remainder of the paper is organized as follows. Section 2 collects together a number of technical results. Section 3 is devoted to the proof of Theorem 1.2. In $\S 4$ the Widder spaces characterized in Theorem 1.2 are identified as spaces of the Laplace-Stieltjes transforms of certain vector-valued measures. Finally, $\S 5$ gives a corollary to Theorem 1.2 that characterizes pseudo-resolvents with values in a Banach algebra, satisfying a growth condition of Hille-Yosida type. Being of significance for the theory of semigroups of operators, the latter result provides motivation for conceiving Theorem 1.2 in the first place.

\section{Auxiliary results}

We begin by establishing a number of technical results that will form a basis for the proof of Theorem 1.2.

Denote by $\|\cdot\|_{\infty}$ the uniform norm $\|f\|_{\infty}=\sup _{t \in \mathbb{R}_{+}}|f(t)|$. For each $\lambda \in \mathbb{R}$, let $C_{\mathrm{b}}\left(\mathbb{R}_{+}, \epsilon_{\lambda}\right)$ be the space of all $\mathbb{F}$-valued continuous functions on $\mathbb{R}_{+}$such that $\epsilon_{\lambda} f$ is bounded. Under the norm $\|f\|_{\infty, \lambda}=\left\|\epsilon_{\lambda} f\right\|_{\infty}, C_{\mathrm{b}}\left(\mathbb{R}_{+}, \epsilon_{\lambda}\right)$ is a Banach space. Note that $\epsilon_{-\mu} \in C_{\mathrm{b}}\left(\mathbb{R}_{+}, \epsilon_{\lambda}\right)$ for each $\mu \geqslant \lambda$, and also $\alpha_{k, \mu} \in C_{\mathrm{b}}\left(\mathbb{R}_{+}, \epsilon_{\lambda}\right)$ for each $k \in \mathbb{N}$ and each $\mu>\lambda$.

The following result is the key to all what ensues next. It is a generalization of a wellknown result from the theory of Borel summability [15, Equation (10.4.13)]. Its proof is 
based on a refinement of a well-known probabilistic argument [12, Chapter VII, Lemma 1 and Equation (1.5)].

Proposition 2.1. Let $\lambda \in \mathbb{R}$ and let $f \in C_{\mathrm{b}}\left(\mathbb{R}_{+}, \epsilon_{\lambda}\right)$. Then, for each $a>0$, the series

$$
\sum_{k=0}^{\infty} f\left(\frac{k}{a}\right) \gamma_{k, a}(t)
$$

converges locally uniformly in $t \in \mathbb{R}_{+}$. Furthermore, for each $\lambda^{\prime}<\lambda$, there exists $a^{\prime}>0$ such that

$$
\sup _{a>a^{\prime}} \sum_{k=0}^{\infty}\left|f\left(\frac{k}{a}\right)\right|\left\|\gamma_{k, a}\right\|_{\infty, \lambda^{\prime}} \leqslant\|f\|_{\infty, \lambda .} .
$$

We also have

$$
f(t)=\lim _{a \rightarrow \infty} \sum_{k=0}^{\infty} f\left(\frac{k}{a}\right) \gamma_{k, a}(t)
$$

for all $t \in \mathbb{R}_{+}$.

Proof. Since, for each $t \in \mathbb{R}_{+}$,

$$
|f(t)| \leqslant\|f\|_{\infty, \lambda} \mathrm{e}^{-\lambda t}
$$

we have

$$
\begin{aligned}
\sum_{k=0}^{\infty}\left|f\left(\frac{k}{a}\right)\right| \gamma_{k, a}(t) & \leqslant\|f\|_{\infty, \lambda} \sum_{k=0}^{\infty} \mathrm{e}^{-\lambda k / a} \frac{(a t)^{k}}{k !} \mathrm{e}^{-a t} \\
& =\|f\|_{\infty, \lambda} \exp \left[a t\left(\mathrm{e}^{-\lambda / a}-1\right)\right]
\end{aligned}
$$

for all $a>0$ and all $t \in \mathbb{R}_{+}$. This immediately implies that, for each $a>0$, the series $\sum_{k=0}^{\infty} f(k / a) \gamma_{k, a}(t)$ converges locally uniformly in $t \in \mathbb{R}_{+}$.

Note that

$$
\lim _{a \rightarrow \infty} a\left(1-\mathrm{e}^{-\lambda / a}\right)=\lambda .
$$

Hence if $\lambda^{\prime}$ satisfies $\lambda^{\prime}<\lambda$, then there exists $a^{\prime}>0$ such that $a\left(\mathrm{e}^{-\lambda / a}-1\right)<-\lambda^{\prime}$ for all $a>a^{\prime}$. Consequently, in view of (2.4),

$$
\sum_{k=0}^{\infty}\left|f\left(\frac{k}{a}\right)\right| \gamma_{k, a}(t) \leqslant\|f\|_{\infty, \lambda} \mathrm{e}^{-\lambda^{\prime} t}
$$

for all $a>a^{\prime}$ and all $t \in \mathbb{R}_{+}$, which gives (2.1).

It remains to prove (2.2). Fix $t \in \mathbb{R}_{+}$arbitrarily. Given $a>0$, let $Y_{a t}$ be a Poisson random variable with parameter $a t$, carried by a probability space $(\Omega, \mathfrak{M}, \mathrm{P})$; that is to say, $Y_{a t}$ is a $\mathbb{Z}^{+}$-valued random variable such that

$$
\mathrm{P}\left[Y_{a t}=k\right]=\frac{(a t)^{k}}{k !} \mathrm{e}^{-a t}=\gamma_{k, a}(t)
$$


for all $k \in \mathbb{Z}^{+}$. Set $X_{a, t}=Y_{a t} / a$. Denoting by $\mathrm{E}[X]$ the expected value of the random variable $X$, we clearly have

$$
\mathrm{E}\left[f\left(X_{a, t}\right)\right]=\sum_{k=0}^{\infty} f\left(\frac{k}{a}\right) \gamma_{k, a}(t)
$$

and further

$$
\mathrm{E}\left[f\left(X_{a, t}\right)-f(t)\right]=\sum_{k=0}^{\infty} f\left(\frac{k}{a}\right) \gamma_{k, a}(t)-f(t) .
$$

Thus, in order to prove (2.2), it suffices to show that

$$
\lim _{a \rightarrow \infty} \mathrm{E}\left[f\left(X_{a, t}\right)-f(t)\right]=0 .
$$

Choose $\epsilon>0$ arbitrarily. Since $f$ is continuous, there exists $\delta>0$ such that $\mid f(x)-$ $f(t) \mid<\epsilon / 2$ for every $x \in \mathbb{R}_{+}$with $|x-t|<\delta$. Let

$$
A_{a, t, \delta}=\left\{\omega \in \Omega|| X_{a, t}(\omega)-t \mid<\delta\right\} .
$$

As is customary, given a set $A$, denote by $1_{A}$ the characteristic function of $A$. Now

$$
\left|\mathrm{E}\left[f\left(X_{a, t}\right)-f(t)\right]\right| \leqslant \mathrm{E}\left[\left|f\left(X_{a, t}\right)-f(t)\right| 1_{A_{a, t, \delta}}\right]+\mathrm{E}\left[\left|f\left(X_{a, t}\right)-f(t)\right| 1_{\Omega \backslash A_{a, t, \delta}}\right] .
$$

Of course, $\left|f\left(X_{a, t}\right)-f(t)\right| 1_{A_{a, t, \delta}} \leqslant(\epsilon / 2) 1_{A_{a, t, \delta}}$, and so

$$
\mathrm{E}\left[\left|f\left(X_{a, t}\right)-f(t)\right| 1_{A_{a, t, \delta}}\right] \leqslant(\epsilon / 2) \mathrm{P}\left[A_{a, t, \delta}\right] \leqslant \epsilon / 2 .
$$

We shall prove shortly that

$$
\lim _{a \rightarrow \infty} \mathrm{E}\left[\left|f\left(X_{a, t}\right)-f(t)\right| 1_{\Omega \backslash A_{a, t, \delta}}\right]=0 .
$$

Assuming this for now, select $a_{0}>0$ so that

$$
\mathrm{E}\left[\left|f\left(X_{a, t}\right)-f(t)\right| 1_{\Omega \backslash A_{a, t, \delta}}\right]<\epsilon / 2
$$

for all $a>a_{0}$. Then, on account of (2.7) and (2.8), $\mathrm{E}\left[\left|f\left(X_{a, t}\right)-f(t)\right|\right]<\epsilon$ for all $a>a_{0}$. We thus see that (2.6) holds.

We proceed to prove (2.9). By the Cauchy-Schwarz inequality,

$$
\begin{aligned}
\mathrm{E}\left[\left|f\left(X_{a, t}\right)-f(t)\right| 1_{\Omega \backslash A_{a, t, \delta}}\right] & \leqslant\left(\mathrm{E}\left[\left|f\left(X_{a, t}\right)-f(t)\right|^{2}\right]\right)^{1 / 2}\left(\mathrm{E}\left[\left(1_{\Omega \backslash A_{a, t, \delta}}\right)^{2}\right]\right)^{1 / 2} \\
& =\left(\mathrm{E}\left[\left|f\left(X_{a, t}\right)-f(t)\right|^{2}\right]\right)^{1 / 2}\left(\mathrm{P}\left[\Omega \backslash A_{a, t, \delta}\right]\right)^{1 / 2} .
\end{aligned}
$$

By the triangle inequality for the $L^{2}$ norm,

$$
\begin{aligned}
\left(\mathrm{E}\left[\left|f\left(X_{a, t}\right)-f(t)\right|^{2}\right]\right)^{1 / 2} & \leqslant\left(\mathrm{E}\left[\left|f\left(X_{a, t}\right)\right|^{2}\right]\right)^{1 / 2}+\left(\mathrm{E}\left[|f(t)|^{2}\right]\right)^{1 / 2} \\
& =\left(\mathrm{E}\left[\left|f\left(X_{a, t}\right)\right|^{2}\right]\right)^{1 / 2}+|f(t)| .
\end{aligned}
$$


Clearly,

$$
\mathrm{E}\left[\left|f\left(X_{a, t}\right)\right|^{2}\right]=\sum_{k=0}^{\infty}\left|f\left(\frac{k}{a}\right)\right|^{2} \gamma_{k, a}(t)
$$

and, by (2.3),

$$
\sum_{k=0}^{\infty}\left|f\left(\frac{k}{a}\right)\right|^{2} \gamma_{k, a}(t) \leqslant\|f\|_{\infty, \lambda}^{2} \sum_{k=0}^{\infty} \mathrm{e}^{-2 \lambda k / a} \gamma_{k, a}(t)=\|f\|_{\infty, \lambda}^{2} \exp \left[a t\left(\mathrm{e}^{-2 \lambda / a}-1\right)\right] .
$$

Since $\lim _{a \rightarrow \infty} a\left(\mathrm{e}^{-2 \lambda / a}-1\right)=-2 \lambda$, it follows that

$$
\limsup _{a \rightarrow \infty} \mathrm{E}\left[\left|f\left(X_{a, t}\right)\right|^{2}\right] \leqslant\|f\|_{\infty, \lambda}^{2} \mathrm{e}^{-2 \lambda t}
$$

and further, by (2.11),

$$
\limsup _{a \rightarrow \infty}\left(\mathrm{E}\left[\left|f\left(X_{a, t}\right)-f(t)\right|^{2}\right]\right)^{1 / 2} \leqslant\|f\|_{\infty, \lambda} \mathrm{e}^{-\lambda t}+|f(t)| .
$$

On the other hand, since

$$
\Omega \backslash A_{a, t, \delta}=\left\{\omega \in \Omega|| X_{a, t}(\omega)-a \mid \geqslant \delta\right\},
$$

an application of Chebyshev's inequality implies that

$$
\mathrm{P}\left[\Omega \backslash A_{a, t, \delta}\right] \leqslant \frac{\mathrm{E}\left[\left(X_{a, t}-t\right)^{2}\right]}{\delta^{2}} .
$$

It is a well-known property of Poisson variables that

$$
\mathrm{E}\left[Y_{a t}\right]=\operatorname{var} Y_{a t}=a t
$$

where var $X$ denotes the variance of the random variable $X$, defined - let us recall - by $\operatorname{var} X=\mathrm{E}\left[|X-\mathrm{E}[X]|^{2}\right]$. Hence

$$
\mathrm{E}\left[X_{a, t}\right]=\frac{\mathrm{E}\left[Y_{a t}\right]}{a}=t
$$

and

$$
\operatorname{var} X_{a, t}=\frac{\operatorname{var} Y_{a t}}{a^{2}}=\frac{t}{a} .
$$

Rewriting the last equality as

$$
\mathrm{E}\left[\left(X_{a, t}-t\right)^{2}\right]=\frac{t}{a}
$$

and combining it with (2.13), we conclude that

$$
\lim _{a \rightarrow \infty} \mathrm{P}\left[\Omega \backslash A_{a, t, \delta}\right]=0 .
$$

This together with (2.10) and (2.12) implies (2.9). 
For each $\lambda \in \mathbb{R}$, let $P\left(\mathbb{R}_{+}, \lambda\right)$ be the linear space spanned by the set $\left\{\epsilon_{-\mu} \mid \mu>\lambda\right\}$. Clearly,

$$
P\left(\mathbb{R}_{+}, \lambda\right)=\bigcup_{\nu>\lambda} P\left(\mathbb{R}_{+}, \nu\right)
$$

Under pointwise multiplication, $P\left(\mathbb{R}_{+}, 0\right)$ is an algebra. Moreover, all the $P\left(\mathbb{R}_{+}, \lambda\right)(\lambda \in$ $\mathbb{R})$ are modules for $P\left(\mathbb{R}_{+}, 0\right)$.

Let $C_{0}\left(\mathbb{R}_{+}\right)$be the space of all $\mathbb{F}$-valued continuous functions $f$ on $\mathbb{R}_{+}$vanishing at infinity.

Proposition 2.2. Let $\mathrm{m}$ be a non-negative Borel measure on $\mathbb{R}_{+}$with bound $\omega$. Then, for each $\mu>\omega, P\left(\mathbb{R}_{+}, \mu\right)$ is dense in $L^{1}\left(\mathbb{R}_{+}, \mathrm{m}\right)$. In particular, $P\left(\mathbb{R}_{+}, \omega\right)$ is dense in $L^{1}\left(\mathbb{R}_{+}, \mathrm{m}\right)$.

Proof. It suffices to prove the first assertion, the other being evident in light of (2.14). Fix $\mu>\omega$ arbitrarily. Let $\mathrm{n}$ be the Borel measure on $\mathbb{R}_{+}$defined by $\mathrm{d} n=\epsilon_{-\mu} \mathrm{dm}$. Clearly, $\mathrm{n}$ is finite. Let $f \in L^{1}\left(\mathbb{R}_{+}, \mathrm{m}\right)$. Then $\epsilon_{\mu} f \in L^{1}\left(\mathbb{R}_{+}, \mathrm{n}\right)$ and, given $\epsilon>0$, there exists $\varphi \in C_{0}\left(\mathbb{R}_{+}\right)$such that $\left\|\epsilon_{\mu} f-\varphi\right\|_{1, \mathrm{n}}<\epsilon / 2$. A standard argument based on the Stone Weierstrass theorem shows that the algebra $P\left(\mathbb{R}_{+}, 0\right)$ is dense in $C_{0}\left(\mathbb{R}_{+}\right)$. Therefore, there exists $p \in P\left(\mathbb{R}_{+}, 0\right)$ such that $\|\varphi-p\|_{\infty}<\epsilon /\left(2\left\|\epsilon_{-\mu}\right\|_{1, \mathrm{~m}}\right)$. Since

$$
\begin{aligned}
\left\|f-\epsilon_{-\mu} p\right\|_{1, \mathrm{~m}} & \leqslant\left\|f-\epsilon_{-\mu} \varphi\right\|_{1, \mathrm{~m}}+\left\|\epsilon_{-\mu} \varphi-\epsilon_{-\mu} p\right\|_{1, \mathrm{~m}}, \\
\left\|f-\epsilon_{-\mu} \varphi\right\|_{1, \mathrm{~m}} & =\left\|\epsilon_{\mu} f-\varphi\right\|_{1, \mathrm{n}}, \\
\left\|\epsilon_{-\mu} \varphi-\epsilon_{-\mu} p\right\|_{1, \mathrm{~m}} & \leqslant\left\|\epsilon_{-\mu}\right\|_{1, \mathrm{~m}}\|\varphi-p\|_{\infty},
\end{aligned}
$$

it follows that $\left\|f-\epsilon_{-\mu} p\right\|_{1, \mathrm{~m}}<\epsilon$. Noting that $\epsilon_{-\mu} p \in P\left(\mathbb{R}_{+}, \mu\right)$ finishes the proof.

Proposition 2.3. Let $\mathrm{m}$ be a non-negative Borel measure on $\mathbb{R}_{+}$with bound $\omega$, let $\lambda>\omega$, and let $f \in C_{\mathrm{b}}\left(\mathbb{R}_{+}, \epsilon_{\lambda}\right)$. Then

$$
\lim _{a \rightarrow \infty} \sum_{k=0}^{\infty}\left|f\left(\frac{k}{a}\right)\right|\left\|\gamma_{k, a}\right\|_{1, \mathrm{~m}}=\|f\|_{1, \mathrm{~m}} .
$$

Proof. Applying the first assertion in Proposition 2.1 with $|f|$ in place of $f$, we deduce that, for each $a>0$, the series

$$
\sum_{k=0}^{\infty}\left|f\left(\frac{k}{a}\right)\right| \gamma_{k, a}(t) \quad\left(t \in \mathbb{R}_{+}\right)
$$

defines a continuous function. Denote this function by $\psi_{a}$. Select $\lambda^{\prime}$ so that $\omega<\lambda^{\prime}<\lambda$. The second assertion in Proposition 2.1 ensures that there exists $a^{\prime}>0$ such that

$$
\left\|\psi_{a}\right\|_{\infty, \lambda^{\prime}} \leqslant\|f\|_{\infty, \lambda}
$$

for all $a>a^{\prime}$. Therefore, for each $a>a^{\prime}, \psi_{a}$ is a function in $C_{\mathrm{b}}\left(\mathbb{R}_{+}, \epsilon_{\lambda^{\prime}}\right)$ and

$$
\psi_{a} \leqslant\|f\|_{\infty, \lambda} \epsilon_{-\lambda^{\prime}} .
$$


To prove (2.15), it suffices to show that

$$
\lim _{n \rightarrow \infty} \sum_{k=0}^{\infty}\left|f\left(\frac{k}{a_{n}}\right)\right|\left\|\gamma_{k, a_{n}}\right\|_{1, \mathrm{~m}}=\|f\|_{1, \mathrm{~m}}
$$

for any sequence $\left\{a_{n}\right\}$ in $\left(a^{\prime}, \infty\right)$ diverging to infinity. Let $\left\{a_{n}\right\}$ be a sequence in $\left(a^{\prime}, \infty\right)$ with $\lim _{n \rightarrow \infty} a_{n}=\infty$. The third assertion in Proposition 2.1 applied to $|f|$ yields

$$
\lim _{n \rightarrow \infty} \psi_{a_{n}}(t)=|f(t)|
$$

for all $t \in \mathbb{R}_{+}$. As $\lambda^{\prime}>\omega$, the function $\epsilon_{-\lambda^{\prime}}$ is m-integrable, and so, in view of (2.16), $\left\{\psi_{a_{n}}\right\}$ has an m-integrable majorant. By Lebesgue's dominated convergence theorem,

$$
\lim _{n \rightarrow \infty} \int_{\mathbb{R}_{+}} \psi_{a_{n}}(t) \mathrm{dm}(t)=\int_{\mathbb{R}_{+}}|f(t)| \operatorname{dm}(t)=\|f\|_{1, \mathrm{~m}} .
$$

On the other hand, an application of Levi's monotone convergence theorem implies that

$$
\begin{aligned}
\int_{\mathbb{R}_{+}} \psi_{a}(t) \mathrm{dm}(t) & =\sum_{k=0}^{\infty} \int_{\mathbb{R}_{+}}\left|f\left(\frac{k}{a}\right)\right| \gamma_{k, a}(t) \mathrm{dm}(t) \\
& =\sum_{k=0}^{\infty}\left|f\left(\frac{k}{a}\right)\right|\left\|\gamma_{k, a}\right\|_{1, \mathrm{~m}}
\end{aligned}
$$

for all $a>0$. Thus (2.17) is established.

Proposition 2.4. Let $\mathrm{m}$ be a non-negative Borel measure on $\mathbb{R}_{+}$with bound $\omega$, and let $E$ be a Banach space. Then any function in $C_{W}^{\infty}((\omega, \infty), E ; \mathrm{m})$ is real analytic.

Proof. Let $r \in C_{W}^{\infty}((\omega, \infty), E ; \mathrm{m})$ and let $\epsilon>0$. If $\xi>\omega+2 \epsilon$, then

$$
\sum_{k=0}^{\infty} \frac{(\epsilon t)^{k}}{k !} \mathrm{e}^{-\xi t}=\mathrm{e}^{-(\xi-\epsilon) t} \leqslant \mathrm{e}^{-(\omega+\epsilon) t}
$$

for all $t \in \mathbb{R}_{+}$. Integrating with respect to the variable $t$ against $\mathrm{m}$, we obtain

$$
\sum_{k=0}^{\infty} \epsilon^{k}\left\|\alpha_{k, \xi}\right\|_{1, \mathrm{~m}} \leqslant\left\|\epsilon_{-(\omega+\epsilon)}\right\|_{1, \mathrm{~m}} .
$$

Hence, for each $k \in \mathbb{Z}^{+}$,

$$
\left\|\alpha_{k, \xi}\right\|_{1, \mathrm{~m}} \leqslant\left\|\epsilon_{-(\omega+\epsilon)}\right\|_{1, \mathrm{~m}} \epsilon^{-k}
$$

and further

$$
\frac{1}{k !}\left\|r^{(k)}(\xi)\right\| \leqslant\|r\|_{W, \mathrm{~m}, \omega}\left\|\epsilon_{-(\omega+\epsilon)}\right\|_{1, \mathrm{~m}} \epsilon^{-k} .
$$

Fix $\lambda>\omega+2 \epsilon$ arbitrarily. Choose $\delta>0$ so that $\lambda-\delta>\omega+2 \epsilon$. If $x \in(\lambda-\delta, \lambda+\delta)$, then, for each $\xi$ belonging to the interval joining $\lambda$ and $x$,

$$
\frac{1}{k !}\left\|r^{(k)}(\xi)\right\||x-\lambda|^{k} \leqslant\|r\|_{W, \mathrm{~m}, \omega}\left\|\epsilon_{-(\omega+\epsilon)}\right\|_{1, \mathrm{~m}}(|x-\lambda| / \epsilon)^{k} .
$$


If $|x-\lambda|<\epsilon^{-1}$, Taylor's formula implies that

$$
\sum_{k=0}^{\infty} \frac{1}{k !} r^{(k)}(\lambda)(x-\lambda)^{k}
$$

converges to $r(x)$. Thus $r$ is real analytic at $\lambda$, and hence is real analytic on all of $(\omega+2 \epsilon, \infty)$. Since $\epsilon$ is arbitrary, the theorem follows.

Proposition 2.5. Let $\mathrm{m}$ be a non-negative Borel measure on $\mathbb{R}_{+}$with bound $\omega$, let $E$ be a Banach space, and let $r \in C_{W}^{\infty}((\omega, \infty), E ; \mathrm{m})$. If $\lambda>\omega$, then there exists $a_{1}>0$ such that

$$
\sum_{k=0}^{\infty} \frac{(-1)^{k}}{k !} a^{k} \mathrm{e}^{-\lambda k / a} r^{(k)}(a)=r\left(a\left(1-\mathrm{e}^{-\lambda / a}\right)\right)
$$

for all $a>a_{1}$.

Proof. Let $\lambda>\omega$. Since $\epsilon_{-\lambda} \in C_{\mathrm{b}}\left(\mathbb{R}_{+}, \epsilon_{\lambda}\right)$, it follows from Proposition 2.3 that there exists $a_{0}>0$ such that

$$
\sum_{k=0}^{\infty} \mathrm{e}^{-\lambda k / a}\left\|\gamma_{k, a}\right\|_{1, \mathrm{~m}}<\infty
$$

for all $a>a_{0}$. Taking into account (2.5), we infer that there exists $a_{0}^{\prime}>0$ such that

$$
a\left(1-\mathrm{e}^{-\lambda / a}\right)>\omega
$$

for all $a>a_{0}^{\prime}$. Let $a_{1}$ be a positive number no smaller than both $a_{0}$ and $a_{0}^{\prime}$. Fix $a>a_{1}$ arbitrarily. Since

$$
\frac{1}{k !} a^{k}\left\|r^{(k)}(a)\right\| \leqslant\|r\|_{W, \mathrm{~m}, \omega}\left\|\gamma_{k, a}\right\|_{1, \mathrm{~m}},
$$

if follows from (2.19) that

$$
\sum_{k=0}^{\infty} \frac{1}{k !} a^{k} \mathrm{e}^{-\lambda k / a}\left\|r^{(k)}(a)\right\|<\infty
$$

for all $a>a_{1}$. Thus the power series

$$
\sum_{k=0}^{\infty} \frac{1}{k !} r^{(k)}(a) t^{k}
$$

converges for all $|t| \leqslant a \mathrm{e}^{-\lambda / a}$. Denote by $\varphi$ the function defined by this series. Clearly, $\varphi$ is real analytic on $I=\left(-a \mathrm{e}^{-\lambda / a}, a \mathrm{e}^{-\lambda / a}\right)$. We claim that $\varphi$ is continuous on $\bar{I}=$ $\left[-a \mathrm{e}^{-\lambda / a}, a \mathrm{e}^{-\lambda / a}\right]$.

It suffices to prove that $\varphi$ is continuous at each endpoint of $\bar{I}$. Let $\sigma \in\{-1,1\}$. Suppose that $t$ satisfies $|t| \leqslant a \mathrm{e}^{-\lambda / a}$ and is so close to $\sigma a \mathrm{e}^{-\lambda / a}$ that $\left|\sigma a \mathrm{e}^{-\lambda / a}-t\right|<a \mathrm{e}^{-\lambda / a} / 2$. Then $\left|a \mathrm{e}^{-\lambda / a}-\sigma t\right|<a \mathrm{e}^{-\lambda / a} / 2$, and so $\sigma t>a \mathrm{e}^{-\lambda / a} / 2$, showing that $\sigma t$ is positive and equal to $|t|$. Now

$$
\left|\left(\sigma t a \mathrm{e}^{-\lambda / a}\right)^{k}-t^{k}\right|=\left|\left(a \mathrm{e}^{-\lambda / a}\right)^{k}-(\sigma t)^{k}\right|=\left(a \mathrm{e}^{-\lambda / a}\right)^{k}-|t|^{k}
$$


for all $k \in \mathbb{Z}^{+}$. Consequently,

$$
\begin{aligned}
\left\|\varphi\left(\sigma a \mathrm{e}^{-\lambda / a}\right)-\varphi(t)\right\| & \leqslant \sum_{k=0}^{\infty} \frac{\left|\left(\sigma t a \mathrm{e}^{-\lambda / a}\right)^{k}-t^{k}\right|}{k !}\left\|r^{(k)}(a)\right\| \\
& =\sum_{k=0}^{\infty} \frac{\left(a \mathrm{e}^{-\lambda / a}\right)^{k}}{k !}\left\|r^{(k)}(a)\right\|-\sum_{k=0}^{\infty} \frac{|t|^{k}}{k !}\left\|r^{(k)}(a)\right\| .
\end{aligned}
$$

By (2.21) and Abel's limit theorem (cf. [20, Chapter III, $\S 2$, Theorem 2.1]), or alternatively by (2.21) and Levi's monotone convergence theorem applied to the counting measure on $\mathbb{Z}^{+}$, the right-hand side above converges to zero as $|t| \rightarrow a \mathrm{e}^{-\lambda / a}$. This implies that $\varphi$ is continuous at $\sigma a \mathrm{e}^{-\lambda / a}$, establishing the claim.

In light of $(2.20)$, the function $\psi(t)=r(a+t)$ is well defined for $t \in \bar{I}$. By Proposition $2.4, \psi$ is real analytic on $I$, and, clearly, has the same derivatives at the value $t=0$ as $\varphi$. Therefore $\varphi=\psi$ on $I$. Since both $\varphi$ and $\psi$ are continuous on $\bar{I}$, they coincide on $\bar{I}$, in particular at the value $t=-a \mathrm{e}^{-\lambda / a}$. This establishes (2.18).

Proposition 2.6. Let $\mathrm{m}$ be a non-negative Borel measure on $\mathbb{R}_{+}$with bound $\omega$. Then the function $(\omega, \infty) \ni \lambda \mapsto \epsilon_{-\lambda} \in L^{1}\left(\mathbb{R}_{+}, \mathrm{m}\right)$ is infinitely differentiable in the norm topology of $L^{1}\left(\mathbb{R}_{+}, \mathrm{m}\right)$, and

$$
\frac{\mathrm{d}^{k} \epsilon_{-\lambda}}{\mathrm{d} \lambda^{k}}=(-1)^{k} \beta_{k, \lambda}
$$

for each $k \in \mathbb{Z}^{+}$and each $\lambda>\omega$.

Proof. Given that $\beta_{0, \lambda}=\epsilon_{-\lambda}$, it suffices to show that, for each $k \in \mathbb{Z}^{+}$, the function $(\omega, \infty) \ni \lambda \mapsto \beta_{k, \lambda} \in L^{1}\left(\mathbb{R}_{+}, \mathrm{m}\right)$ is differentiable in the norm topology of $L^{1}\left(\mathbb{R}_{+}, \mathrm{m}\right)$ and

$$
\frac{\mathrm{d} \beta_{k, \lambda}}{\mathrm{d} \lambda}=-\beta_{k+1, \lambda}
$$

Fix $k \in \mathbb{Z}^{+}$arbitrarily. Direct computation shows that

$$
\frac{\partial \beta_{k, \lambda}}{\partial \lambda}(t)=-\beta_{k+1, \lambda}(t)
$$

for all $\lambda, t \in \mathbb{R}$. Fix $\lambda>\omega$ arbitrarily, and next select $\omega^{\prime}$ and $\omega^{\prime \prime}$ so that $\omega<\omega^{\prime}<\omega^{\prime \prime}<\lambda$. Let $h$ be such that $0<|h|<\omega^{\prime \prime}-\omega^{\prime}$. Taking into account (2.22) and Taylor's formula, we see that, given $t \in \mathbb{R}_{+}$, there exists $\xi_{t}$ in the interval joining $\lambda$ and $\lambda+h$ such that

$$
\frac{\beta_{k, \lambda+h}(t)-\beta_{k, \lambda}(t)}{h}=-\beta_{k+1, \xi_{t}}(t) .
$$

Clearly, $\beta_{k+1, \lambda} \in C_{\mathrm{b}}\left(\mathbb{R}_{+}, \omega^{\prime \prime}\right)$ and

$$
t^{k+1} \mathrm{e}^{-\lambda t} \leqslant\left\|\beta_{k+1, \lambda}\right\|_{\infty, \omega^{\prime \prime}} \mathrm{e}^{-\omega^{\prime \prime} t} .
$$

Hence

$$
\begin{aligned}
\beta_{k+1, \xi_{t}}(t) & =t^{k+1} \mathrm{e}^{-\xi_{t} t} \leqslant t^{k+1} \mathrm{e}^{-\lambda t} \mathrm{e}^{|h| t} \leqslant\left\|\beta_{k+1, \lambda}\right\|_{\infty, \omega^{\prime \prime}} \mathrm{e}^{-\omega^{\prime \prime} t} \mathrm{e}^{\left(\omega^{\prime \prime}-\omega^{\prime}\right) t} \\
& =\left\|\beta_{k+1, \lambda}\right\|_{\infty, \omega^{\prime \prime}} \mathrm{e}^{-\omega^{\prime} t}
\end{aligned}
$$


showing that the functions

$$
t \mapsto \frac{\beta_{k, \lambda+h}(t)-\beta_{k, \lambda}(t)}{h} \quad\left(0<|h|<\omega^{\prime \prime}-\omega^{\prime}\right)
$$

are dominated by an m-integrable function. Let $\left\{h_{n}\right\}$ be an arbitrary sequence in $\left(0, \omega^{\prime \prime}-\right.$ $\left.\omega^{\prime}\right)$ tending to zero as $n \rightarrow \infty$. Since, by $(2.22)$, the sequence $\left\{\left(\beta_{k, \lambda+h_{n}}-\beta_{k, \lambda}\right) / h_{n}\right\}$ tends pointwise to $-\beta_{k+1, \lambda}$ as $n \rightarrow \infty$, an appeal to Lebesgue's dominated convergence theorem reveals that

$$
\lim _{n \rightarrow \infty}\left\|\frac{\beta_{k, \lambda+h_{n}}-\beta_{k, \lambda}}{h_{n}}+\beta_{k+1, \lambda}\right\|_{1, \mathrm{~m}}=0
$$

which is the desired result.

\section{Proof of the main result}

Proof of Theorem 1.2. Let $r:(\omega, \infty) \rightarrow E$ be a function such that $r(\lambda)=T\left(\epsilon_{-\lambda}\right)$ for some bounded linear operator $T: L^{1}\left(\mathbb{R}_{+}, \mathrm{m}\right) \rightarrow E$ and all $\lambda>\omega$. Since, according to Proposition $2.2, P\left(\mathbb{R}_{+}, \omega\right)$ is dense in $L^{1}\left(\mathbb{R}_{+}, \mathrm{m}\right), T$ is uniquely determined by its values taken on at the $\epsilon_{-\lambda}(\lambda>\omega)$. By Proposition 2.6, the function $(\omega, \infty) \ni \lambda \mapsto \epsilon_{-\lambda} \in$ $L^{1}\left(\mathbb{R}_{+}, \mathrm{m}\right)$ is infinitely differentiable in the norm topology of $L^{1}\left(\mathbb{R}_{+}, \mathrm{m}\right)$, and

$$
\frac{\mathrm{d}^{k} \epsilon_{-\lambda}}{\mathrm{d} \lambda^{k}}=(-1)^{k} \beta_{k, \lambda}
$$

for each $k \in \mathbb{Z}^{+}$and each $\lambda>\omega$. Therefore $r$ is infinitely differentiable and

$$
T\left(\beta_{k, \lambda}\right)=(-1)^{k} r^{(k)}(\lambda)
$$

for each $k \in \mathbb{Z}^{+}$and each $\lambda>\omega$. Consequently, we have $\|r\|_{W, \mathrm{~m}, \omega} \leqslant\|T\|$, and in particular $\|r\|_{W, \mathbf{m}, \omega}$ is finite. Now-as a moment's reflection reveals - to complete the proof, it suffices to show that if $r$ is a function in $C_{W}^{\infty}((\omega, \infty), E ; \mathrm{m})$, then there exists a bounded linear operator $T: L^{1}\left(\mathbb{R}_{+}, \mathrm{m}\right) \rightarrow E$ such that $T\left(\epsilon_{-\lambda}\right)=r(\lambda)$ for each $\lambda>\omega$ and $\|T\| \leqslant\|r\|_{W, \mathrm{~m}, \omega}$.

Suppose then that $r \in C_{W}^{\infty}((\omega, \infty), E ; \mathrm{m})$. Define a linear operator $T: P\left(\mathbb{R}_{+}, \omega\right) \rightarrow E$ as follows: for each $f \in P\left(\mathbb{R}_{+}, \omega\right)$ representable as

$$
f=\sum_{\lambda \in \Lambda} a_{\lambda} \epsilon_{-\lambda} \quad\left(a_{\lambda} \in \mathbb{F}\right),
$$

where $\Lambda$ is a finite subset of $(\omega, \infty)$, let

$$
T(f)=\sum_{\lambda \in \Lambda} a_{\lambda} r(\lambda) .
$$

Clearly, $T\left(\epsilon_{-\lambda}\right)=r_{\lambda}$ for all $\lambda>\omega$. We shall prove that

$$
\|T(f)\| \leqslant\|r\|_{W, \mathrm{~m}, \omega}\|f\|_{1, \mathrm{~m}} .
$$


This will guarantee that $T$ is well defined and also that $T$ is bounded with $\|T\| \leqslant\|r\|_{W, \mathbf{m}, \omega}$ provided that $P\left(\mathbb{R}_{+}, \omega\right)$ is considered with the norm $\|\cdot\|_{1, \mathrm{~m}}$. Since $P\left(\mathbb{R}_{+}, \omega\right)$ is dense in $L^{1}\left(\mathbb{R}_{+}, \mathrm{m}\right), T$ can next be extended by continuity to a bounded linear operator from $L^{1}\left(\mathbb{R}_{+}, \mathrm{m}\right)$ to $E$, the norm of the extension being equal to $\|T\|$.

In order to prove (3.1), we introduce, for each $n \in \mathbb{N}$ and each $a>0$, a linear operator $T_{n, a}: P\left(\mathbb{R}_{+}, \omega\right) \rightarrow E$ defined by

$$
T_{n, a}(f)=\sum_{k=0}^{n} \frac{(-1)^{k}}{k !} a^{k} f\left(\frac{k}{a}\right) r^{(k)}(a) \quad\left(f \in P\left(\mathbb{R}_{+}, \omega\right)\right) .
$$

Fix $f=\sum_{\lambda \in \Lambda} a_{\lambda} \epsilon_{-\lambda}$ arbitrarily and choose $\mu>\omega$ so that $\Lambda \subset(\mu, \infty)$. Clearly, $\epsilon_{-\lambda} \in$ $C_{\mathrm{b}}\left(\mathbb{R}_{+}, \epsilon_{\mu}\right)$ for all $\lambda \in \Lambda$. By Proposition 2.5, there exists $a_{1}>0$ such that

$$
\lim _{n \rightarrow \infty} T_{n, a}\left(\epsilon_{-\lambda}\right)=r\left(a\left(1-\mathrm{e}^{-\lambda / a}\right)\right)
$$

for all $\lambda \in \Lambda$ and all $a>a_{1}$. Hence

$$
\lim _{n \rightarrow \infty} T_{n, a}(f)=\sum_{\lambda \in \Lambda} a_{\lambda} r\left(a\left(1-\mathrm{e}^{-\lambda / a}\right)\right)
$$

for all $a>a_{1}$. Since $r$ is continuous, it follows from (2.5) that

$$
\lim _{a \rightarrow \infty} r\left(a\left(1-\mathrm{e}^{-\lambda / a}\right)\right)=r(\lambda) .
$$

Consequently,

$$
\lim _{\substack{a \rightarrow \infty \\ a>a_{1}}}\left[\lim _{n \rightarrow \infty} T_{n, a}(f)\right]=T(f) .
$$

Since, for each $n \in \mathbb{N}$ and each $a>0$,

$$
\begin{aligned}
\left\|T_{n, a}(f)\right\| & \leqslant \sum_{k=0}^{n} \frac{1}{k !}\left|f\left(\frac{k}{a}\right)\right| a^{k}\left\|r^{(k)}(a)\right\| \leqslant\|r\|_{W, \mathrm{~m}, \omega} \sum_{k=0}^{n}\left|f\left(\frac{k}{a}\right)\right|\left\|\gamma_{k, a}\right\|_{1, \mathrm{~m}} \\
& \leqslant\|r\|_{W, \mathrm{~m}, \omega} \sum_{k=0}^{\infty}\left|f\left(\frac{k}{a}\right)\right|\left\|\gamma_{k, a}\right\|_{1, \mathrm{~m}},
\end{aligned}
$$

invoking Proposition 2.3 we conclude that

$$
\limsup _{a \rightarrow \infty}\left[\limsup _{n \rightarrow \infty}\left\|T_{n, a}(f)\right\|\right] \leqslant\|r\|_{W, \mathrm{~m}, \omega}\|f\|_{1, \mathrm{~m}} .
$$

This together with (3.2) implies (3.1).

\section{A link with the Laplace-Stieltjes transform}

Let $\mathrm{m}$ be a non-negative Borel measure on $\mathbb{R}_{+}$, and let $E$ be a Banach space. Here we identify Widder spaces of the form $C_{W}^{\infty}((\omega, \infty), E ; \mathrm{m})$, where $\omega$ is a bound for $\mathrm{m}$, as spaces of the Laplace-Stieltjes transforms of certain $E$-valued measures. This characterization 
is based on the fact that the action of operators in $\mathcal{L}\left(L^{1}\left(\mathbb{R}_{+}, \mathrm{m}\right), E\right)$ can be expressed in terms of integrals with respect to $E$-valued measures.

We begin by recalling that a ring, or a clan, of subsets of a set $X$ is a non-empty collection of subsets of $X$ that is closed under pairwise unions and relative complementation (cf. $\left[\mathbf{9}\right.$, p. 1], [13, p. 19]). Let $\mathcal{R}\left(\mathbb{R}_{+}, \mathrm{m}\right)$ be the ring of $\mathrm{m}$-measurable subsets $A$ of $\mathbb{R}_{+}$ such that $\mathrm{m}(A)<\infty$. Note that $\mathcal{R}\left(\mathbb{R}_{+}, \mathrm{m}\right)$ is not an algebra of sets unless $\mathrm{m}\left(\mathbb{R}_{+}\right)<\infty$. Let $M\left(\mathbb{R}_{+}, E ; \mathrm{m}\right)$ be the space of all $E$-valued measures $\boldsymbol{\mu}$ on $\mathcal{R}\left(\mathbb{R}_{+}, \mathrm{m}\right)$ for which there exists a non-negative $C=C(\boldsymbol{\mu})$ such that $\|\boldsymbol{\mu}(A)\| \leqslant C \mathrm{~m}(A)$ for all $A \in \mathcal{R}\left(\mathbb{R}_{+}, \mathrm{m}\right)$. The $\sigma$-additivity of $\mathrm{m}$ immediately implies that every measure in $M\left(\mathbb{R}_{+}, E ; \mathrm{m}\right)$ is $\sigma$-additive. Equipped with the norm

$$
\begin{aligned}
\|\boldsymbol{\mu}\|_{\infty, \mathrm{m}} & =\sup \left\{C \in \mathbb{R}_{+} \mid\|\boldsymbol{\mu}(A)\| \leqslant C \mathrm{~m}(A) \text { for all } A \in \mathcal{R}\left(\mathbb{R}_{+}, \mathrm{m}\right)\right\} \\
& =\sup \left\{\|\boldsymbol{\mu}(A)\| / \mathrm{m}(A) \mid A \in \mathcal{R}\left(\mathbb{R}_{+}, \mathrm{m}\right) \text { with } \mathrm{m}(A)>0\right\},
\end{aligned}
$$

$M\left(\mathbb{R}_{+}, E ; \mathrm{m}\right)$ is a Banach space. As it turns out, the spaces $\mathcal{L}\left(L^{1}\left(\mathbb{R}_{+}, \mathrm{m}\right), E\right)$ and $M\left(\mathbb{R}_{+}, E ; \mathrm{m}\right)$ are isometrically isomorphic. Below we outline the proof of this result.

Let $T \in \mathcal{L}\left(L^{1}\left(\mathbb{R}_{+}, \mathrm{m}\right), E\right)$ be a continuous linear operator. For each $A \in \mathcal{R}\left(\mathbb{R}_{+}, \mathrm{m}\right)$, define $\boldsymbol{\mu}_{T}(A)$ to be $T\left(1_{A}\right)$. The set function $\boldsymbol{\mu}_{T}: \mathcal{R}\left(\mathbb{R}_{+}, \mathrm{m}\right) \rightarrow E, A \mapsto \boldsymbol{\mu}_{T}(A)$, is easily seen to be a measure in $M\left(\mathbb{R}_{+}, E ; \mathrm{m}\right)$ satisfying $\left\|\boldsymbol{\mu}_{T}\right\|_{\infty, \mathrm{m}} \leqslant\|T\|$. Accordingly, the mapping $\mathcal{L}\left(L^{1}\left(\mathbb{R}_{+}, \mathrm{m}\right), E\right) \ni T \mapsto \boldsymbol{\mu}_{T} \in M\left(\mathbb{R}_{+}, E ; \mathrm{m}\right)$ is a linear contraction. We proceed to define an inverse map. Let $\mathcal{S}\left(\mathbb{R}_{+}, \mathrm{m}\right)$ be the set of all simple functions from $\mathbb{R}_{+}$into $\mathbb{F}$ of the form $\sum_{i=1}^{n} a_{i} 1_{A_{i}}$, where $a_{i} \in \mathbb{F}$ and $A_{i} \in \mathcal{R}\left(\mathbb{R}_{+}, \mathrm{m}\right)$ for each $i=1, \ldots, n$. Clearly, $\mathcal{S}\left(\mathbb{R}_{+}, \mathrm{m}\right)$ is a dense linear subspace of $L^{1}\left(\mathbb{R}_{+}, \mathrm{m}\right)$. Fix $\boldsymbol{\mu} \in M\left(\mathbb{R}_{+}, E ; \mathrm{m}\right)$ arbitrarily. For each $f \in \mathcal{S}\left(\mathbb{R}_{+}, \mathrm{m}\right)$, let

$$
T_{\boldsymbol{\mu}}(f)=\sum_{a \in \mathbb{F}} a \boldsymbol{\mu}\left(f^{-1}(\{a\})\right) .
$$

A routine verification shows that $\mathcal{S}\left(\mathbb{R}_{+}, \mathrm{m}\right) \ni f \mapsto T_{\boldsymbol{\mu}}(f) \in E$ is a continuous linear operator with norm not greater than $\|\boldsymbol{\mu}\|_{\infty, \mathrm{m}}$. This operator can further be extended by continuity to a continuous linear operator $T_{\mu}: L^{1}\left(\mathbb{R}_{+}, \mathrm{m}\right) \rightarrow E$ with norm not greater than $\|\boldsymbol{\mu}\|_{\infty, \mathrm{m}}$. Clearly, $M\left(\mathbb{R}_{+}, E ; \mathrm{m}\right) \ni \boldsymbol{\mu} \mapsto T_{\boldsymbol{\mu}} \in \mathcal{L}\left(L^{1}\left(\mathbb{R}_{+}, \mathrm{m}\right), E\right)$ is a linear contraction. It is readily verified that the mappings $T \mapsto \boldsymbol{\mu}_{T}$ and $\boldsymbol{\mu} \mapsto T_{\boldsymbol{\mu}}$ are mutually inverse and as such determine an isometric isomorphism between $\mathcal{L}\left(L^{1}\left(\mathbb{R}_{+}, \mathrm{m}\right), E\right)$ and $M\left(\mathbb{R}_{+}, E ; \mathrm{m}\right)$.

Incidentally, note that if $f \in L^{1}\left(\mathbb{R}_{+}, \mathrm{m}\right)$ and $\boldsymbol{\mu} \in M\left(\mathbb{R}_{+}, E ; \mathrm{m}\right)$, then the element $T_{\boldsymbol{\mu}}(f)$ of $E$ coincides with the integral of $f$ with respect to $\boldsymbol{\mu}$ (see [8, pp. 5-6]). Therefore $T_{\boldsymbol{\mu}}(f)$ can also be written as $\int_{\mathbb{R}_{+}} f \mathrm{~d} \boldsymbol{\mu}$.

The space $M\left(\mathbb{R}_{+}, E ; \mathrm{m}\right)$ can be further characterized for $E$ having the Radon-Nikodym property or RNP. Recall that a Banach space $E$ has the RNP provided that if $\boldsymbol{\nu}$ is an $E$-valued measure of finite variation and $\nu$ is absolutely continuous with respect to a finite scalar measure $\mathrm{n}$, then there is an $E$-valued measurable function $g$ so that $\boldsymbol{\nu}(A)=\int_{A} g \mathrm{dn}$ for every measurable set $A$, the integral being taken in the sense of Bochner. An equivalent requirement is that every Lipschitz function from $\mathbb{R}$ into $E$ be differentiable almost everywhere with respect to Lebesgue measure. The RNP is a hereditary property (i.e. passes to closed subspaces) and is enjoyed (amongst others) by 
any reflexive space, any separable dual space, and any $\ell^{1}(\Gamma)$ space, where $\Gamma$ is a set (see $[\mathbf{8}$, Chapter III]).

Let $L^{\infty}\left(\mathbb{R}_{+}, E ; \mathrm{m}\right)$ be the space of (equivalence classes of) $E$-valued $\mathrm{m}$-measurable essentially bounded functions on $\mathbb{R}_{+}$, with the norm

$$
\|f\|_{\infty, \mathrm{m}}=\inf \left\{\sup \{\|f(t)\| \mid t \in A\} \mid A \subset \mathbb{R}_{+} \text {m-measurable with } \mathrm{m}\left(\mathbb{R}_{+} \backslash A\right)=0\right\} .
$$

If $E$ has the RNP, then the spaces $L^{\infty}\left(\mathbb{R}_{+}, E ; \mathrm{m}\right)$ and $M\left(\mathbb{R}_{+}, E ; \mathrm{m}\right)$ are isometrically isomorphic under the correspondence $g \leftrightarrow \boldsymbol{\mu}$, where $g \in L^{\infty}\left(\mathbb{R}_{+}, E ; \mathrm{m}\right)$ and $\boldsymbol{\mu} \in$ $M\left(\mathbb{R}_{+}, E ; \mathrm{m}\right)$, defined by

$$
\boldsymbol{\mu}(B)=\int_{B} g \mathrm{dm} \quad\left(B \in \mathcal{R}\left(\mathbb{R}_{+}, \mathrm{m}\right)\right)
$$

where the integral is taken in the sense of Bochner (see [8, Chapter III]). Consequently, if $(g, \boldsymbol{\mu})$ is a pair of corresponding elements of $L^{\infty}\left(\mathbb{R}_{+}, E ; \mathrm{m}\right)$ and $M\left(\mathbb{R}_{+}, E ; \mathrm{m}\right)$, then any integral of the form

$$
\int_{\mathbb{R}_{+}} f \mathrm{~d} \boldsymbol{\mu} \quad\left(f \in L^{1}\left(\mathbb{R}_{+}, \mathrm{m}\right)\right)
$$

can be represented as the Bochner integral

$$
\int_{\mathbb{R}_{+}} f \mathrm{~d} \boldsymbol{\mu}=\int_{\mathbb{R}_{+}} f g \mathrm{dm} .
$$

In light of the comments above, we can now formulate two immediate corollaries to Theorem 1.2. The first identifies any $C_{W}^{\infty}((\omega, \infty), E ; \mathrm{m})$, where $\omega$ is a bound for $\mathrm{m}$, as the space of the Laplace-Stieltjes transforms on $(\omega, \infty)$ of measures in $M\left(\mathbb{R}_{+}, E ; \mathrm{m}\right)$, whereas the other, under the assumption that $E$ has the RNP, identifies any $C_{W}^{\infty}((\omega, \infty), E ; \mathrm{m})$ as the space of the Laplace-Stieltjes transforms on $(\omega, \infty)$ of $E$-valued measures on $\mathcal{R}\left(\mathbb{R}_{+}, \mathrm{m}\right)$ absolutely continuous with respect to $\mathrm{m}$ with density in $L^{\infty}\left(\mathbb{R}_{+}, E ; \mathrm{m}\right)$.

Theorem 4.1. Let $\mathrm{m}$ be a non-negative Borel measure on $\mathbb{R}_{+}$with bound $\omega$, let $E$ be a Banach space, and let $r:(\omega, \infty) \rightarrow E$ be a function. Then $r$ can be represented in the form

$$
r(\lambda)=\int_{\mathbb{R}_{+}} \epsilon_{-\lambda} \mathrm{d} \boldsymbol{\mu} \quad(\lambda>\omega)
$$

for some $\boldsymbol{\mu} \in M\left(\mathbb{R}_{+}, E ; \mathrm{m}\right)$ if and only if $r \in C_{W}^{\infty}((\omega, \infty), E ; \mathrm{m})$. If there exists a $\boldsymbol{\mu} \in$ $M\left(\mathbb{R}_{+}, E ; \mathrm{m}\right)$ such that $r(\lambda)=\int_{\mathbb{R}_{+}} \epsilon_{-\lambda} \mathrm{d} \boldsymbol{\mu}$ for all $\lambda>\omega$, then $\boldsymbol{\mu}$ is unique and $\|\boldsymbol{\mu}\|_{\infty, \mathrm{m}}=$ $\|r\|_{W, \mathrm{~m}, \omega}$.

Theorem 4.2. Let $\mathrm{m}$ be a non-negative Borel measure on $\mathbb{R}_{+}$with bound $\omega$, let $E$ be a Banach space with the RNP, and let $r:(\omega, \infty) \rightarrow E$ be a function. Then $r$ can be represented in the form

$$
r(\lambda)=\int_{\mathbb{R}_{+}} \epsilon_{-\lambda} g \mathrm{dm} \quad(\lambda>\omega)
$$


for some $g \in L^{\infty}\left(\mathbb{R}_{+}, E ; \mathrm{m}\right)$ if and only if $r \in C_{W}^{\infty}((\omega, \infty), E ; \mathrm{m})$. If there exists a $g \in$ $L^{\infty}\left(\mathbb{R}_{+}, E ; \mathrm{m}\right)$ such that $r(\lambda)=\int_{\mathbb{R}_{+}} \epsilon_{-\lambda} g \mathrm{dm}$ for all $\lambda>\omega$, then $g$ is unique and $\|g\|_{\infty, \mathrm{m}}=$ $\|r\|_{W, \mathrm{~m}, \omega}$.

One more comment is in order. If $\boldsymbol{\mu}$ is a measure in $M\left(\mathbb{R}_{+}, E ; \mathrm{m}\right)$, where $E$ is a Banach space and $m$ is a non-negative Borel measure on $\mathbb{R}_{+}$with bound $\omega$, then, for every $\lambda>\max \{\omega, 0\}$, the Laplace-Stieltjes transform $\int_{\mathbb{R}_{+}} \epsilon_{-\lambda} \mathrm{d} \boldsymbol{\mu}$ can be represented as the Laplace-Carson transform, or $\lambda$-multiplied Laplace transform

$$
\int_{\mathbb{R}_{+}} \epsilon_{-\lambda} \mathrm{d} \boldsymbol{\mu}=\lambda \int_{0}^{\infty} \mathrm{e}^{-\lambda t} g_{\boldsymbol{\mu}}(t) \mathrm{d} t
$$

where $g_{\boldsymbol{\mu}}$ is the function from $\mathbb{R}_{+}$into $E$ given by $g_{\boldsymbol{\mu}}(t)=\boldsymbol{\mu}([0, t])$ for all $t \in \mathbb{R}_{+}$, and the right-hand side integral is taken in the sense of Bochner. To prove this assertion, define $f: \mathbb{R}_{+} \rightarrow L^{1}\left(\mathbb{R}_{+}, \mathrm{m}\right)$ by $f(t)=1_{[0, t]}$ for all $t \in \mathbb{R}_{+}$. We shall show that $f$ is (strongly) Borel measurable. Let $A=\left\{t \in \mathbb{R}_{+}: \mathrm{m}(\{t\})>0\right\}$. Since $\mathrm{m}$ is finite on every bounded Borel subset of $\mathbb{R}_{+}, A$ is at most countable. Let $A=\left\{a_{n}: n \in \mathbb{N}\right\}$ be an enumeration of $A$. Write $f$ as $f=f_{1}+f_{2}$, where $f_{1}$ and $f_{2}$ are the functions from $\mathbb{R}_{+}$ into $L^{1}\left(\mathbb{R}_{+}, \mathrm{m}\right)$ defined by $f_{1}(t)=1_{[0, t] \cap A}$ and $f_{2}(t)=1_{[0, t] \cap\left(\mathbb{R}_{+} \backslash A\right)}$ for all $t \in \mathbb{R}_{+}$. Given $n \in \mathbb{N}$, define $h_{n}: \mathbb{R}_{+} \rightarrow L^{1}\left(\mathbb{R}_{+}, \mathrm{m}\right)$ by $h_{n}(t)=1_{[0, t] \cap\left\{a_{n}\right\}}$ for all $t \in \mathbb{R}_{+}$. Each $h_{n}$ is Borel measurable, being equal to zero on $\left[0, a_{n}\right)$ and to $1_{\left\{a_{n}\right\}}$ on $\left[a_{n}, \infty\right)$. It is readily seen that, for each $t \in \mathbb{R}_{+}, f_{1}(t)=\sum_{n \in \mathbb{N}} h_{n}(t)$, the series being absolutely convergent in the norm topology of $L^{1}\left(\mathbb{R}_{+}, \mathrm{m}\right)$. This implies that $f_{1}$ is Borel measurable. On the other hand, since $\mathrm{m}$ restricted to $\mathbb{R}_{+} \backslash A$ is continuous, it follows that $f_{2}$ is continuous and hence Borel measurable. Thus $f$ is Borel measurable. As an immediate consequence, we see that, for every $\lambda \in \mathbb{R}$, the function $\mathbb{R}_{+} \ni t \mapsto \lambda \mathrm{e}^{-\lambda t} 1_{[0, t]} \in L^{1}\left(\mathbb{R}_{+}, \mathrm{m}\right)$ is Borel measurable. Now, for each $\lambda>0$ and each $s \in \mathbb{R}_{+}$,

$$
\lambda \int_{0}^{\infty} \mathrm{e}^{-\lambda t} 1_{[0, t]}(s) \mathrm{d} t=\lambda \int_{s}^{\infty} \mathrm{e}^{-\lambda t} \mathrm{~d} t=\mathrm{e}^{-\lambda s}
$$

and further, for every $\lambda>\max \{\omega, 0\}$,

$$
\begin{aligned}
\lambda \int_{0}^{\infty} \mathrm{e}^{-\lambda t}\left\|1_{[0, t]}\right\|_{1, \mathrm{~m}} \mathrm{~d} t & =\lambda \int_{0}^{\infty} \mathrm{e}^{-\lambda t}\left[\int_{\mathbb{R}_{+}} 1_{[0, t]}(s) \mathrm{dm}(s)\right] \mathrm{d} t \\
& =\int_{\mathbb{R}_{+}}\left[\lambda \int_{0}^{\infty} \mathrm{e}^{-\lambda t} 1_{[0, t]}(s) \mathrm{d} t\right] \mathrm{dm}(s) \\
& =\int_{\mathbb{R}_{+}} \mathrm{e}^{-\lambda s} \mathrm{dm}(s)<+\infty
\end{aligned}
$$

by Fubini's theorem. Fix $\lambda>\max \{\omega, 0\}$ arbitrarily. According to Bochner's integrability criterion (cf. [23, Chapter $5, \S 5$, Theorem 1]), the function $\mathbb{R}_{+} \ni t \mapsto \lambda \mathrm{e}^{-\lambda t} 1_{[0, t]} \in$ $L^{1}\left(\mathbb{R}_{+}, \mathrm{m}\right)$ is Bochner integrable with respect to Lebesgue measure on $\mathbb{R}_{+}$. In view of (4.2), the Bochner integral $\int_{0}^{\infty} \lambda \mathrm{e}^{-\lambda t} 1_{[0, t]} \mathrm{d} t$ is the element of $L^{1}\left(\mathbb{R}_{+}, \mathrm{m}\right)$ represented by $\epsilon_{-\lambda}$. Now, by the continuity of $T_{\boldsymbol{\mu}}$ (as an operator from $L^{1}\left(\mathbb{R}_{+}, \mathrm{m}\right)$ to $E$ ), the function 
$\mathbb{R}_{+} \ni t \mapsto \lambda \mathrm{e}^{-\lambda t} T_{\boldsymbol{\mu}}\left(1_{[0, t]}\right) \in E$ is Bochner integrable and

$$
T_{\boldsymbol{\mu}}\left(\epsilon_{-\lambda}\right)=\int_{0}^{\infty} \lambda \mathrm{e}^{-\lambda t} T_{\boldsymbol{\mu}}\left(1_{[0, t]}\right) \mathrm{d} t .
$$

Taking into account that $g_{\boldsymbol{\mu}}(t)=T_{\boldsymbol{\mu}}\left(1_{[0, t]}\right)$ for each $t \in \mathbb{R}_{+}$, we finally obtain (4.1).

\section{An application to pseudo-resolvents}

Here we give one application of Theorem 1.2 of importance for operator semigroup theory.

Let $\boldsymbol{A}$ be a Banach algebra (with identity or not) and let $U$ be a subset of $\mathbb{F}$. A function $r: U \rightarrow \boldsymbol{A}, \lambda \mapsto r_{\lambda}$, is called a pseudo-resolvent if it satisfies the following Hilbert equation:

$$
r_{\lambda}-r_{\mu}=(\mu-\lambda) r_{\lambda} r_{\mu} \quad(\lambda, \mu \in U) .
$$

Let $U$ be an open subset of $\mathbb{R}$ and let $r: U \rightarrow \boldsymbol{A}$ be a pseudo-resolvent. It is well known that $r$ is then infinitely differentiable, in fact real analytic, and

$$
\frac{\mathrm{d}^{k} r_{\lambda}}{\mathrm{d} \lambda^{k}}=(-1)^{k} k ! r_{\lambda}^{k+1}
$$

for all $k \in \mathbb{Z}^{+}$(cf. [11, Chapter IX, $\left.\S 1\right],[\mathbf{1 5}, \S 5.8],[\mathbf{2 3}$, Chapter VIII, $\S 4]$ ). Thus, if $\mathrm{m}$ is a non-negative Borel measure on $\mathbb{R}_{+}$with bound $\omega$ and $U=(\omega, \infty)$, then

$$
\|r\|_{W, \mathbf{m}, \omega}=\sup \left\{\left\|r_{\lambda}^{k+1}\right\| /\left\|\alpha_{k, \lambda}\right\|_{1, \mathrm{~m}} \mid k \in \mathbb{Z}^{+}, \lambda \in(\omega, \infty)\right\} .
$$

When $\mathrm{m}$ has a density $\Omega$ (with respect to Lebesgue measure) of the form $\Omega(t)=\mathrm{e}^{\omega t}$ $\left(t \in \mathbb{R}_{+}, \omega \in \mathbb{R}\right)$, we have $\left\|\alpha_{k, \lambda}\right\|_{1, \mathrm{~m}}=(\lambda-\omega)^{-(k+1)}$ for all $k \in \mathbb{Z}^{+}$and all $\lambda>\omega$, and the equality above reduces to

$$
\|r\|_{W, \Omega, \omega}=\sup \left\{(\lambda-\omega)^{k}\left\|r_{\lambda}^{k}\right\| \mid k \in \mathbb{N}, \lambda \in(\omega, \infty)\right\} .
$$

Now observe that $\|r\|_{W, \Omega, \omega}<\infty$ is nothing else but the Hille-Yosida condition intervening in the Hille-Yosida theorem on the generation of one-parameter semigroups of operators (cf. [10, Chapter VIII, $\S 1$, Theorem 13], [15, Theorem 12.3.1], [23, Chapter 9, $\S 7])$. By analogy, when $\mathrm{m}$ is a non-negative Borel measure on $\mathbb{R}_{+}$with bound $\omega$ and $r:(\omega, \infty) \rightarrow \boldsymbol{A}$ is a pseudo-resolvent, $r$ will be said to satisfy the Hille-Yosida condition if $\|r\|_{W, \mathrm{~m}, \omega}<\infty$.

Direct verification shows that if $\Omega$ is a weight function on $\mathbb{R}_{+}$with bound $\omega$, then $\epsilon:(\omega, \infty) \rightarrow L^{1}\left(\mathbb{R}_{+}, \Omega\right), \lambda \mapsto \epsilon_{-\lambda}$, is a pseudo-resolvent with $\|\epsilon\|_{W, \Omega, \omega}=1$. As will become clear in a moment, from a certain point of view $\epsilon$ can be regarded as a canonical pseudo-resolvent in $L^{1}\left(\mathbb{R}_{+}, \Omega\right)$.

Let $\Omega$ be a weight function on $\mathbb{R}_{+}$with bound $\omega$, suppose that $r:(\omega, \infty) \rightarrow \boldsymbol{A}, \lambda \mapsto r_{\lambda}$, is a pseudo-resolvent, and let $T: L^{1}\left(\mathbb{R}_{+}, \Omega\right) \rightarrow \boldsymbol{A}$ be a bounded linear operator such that $r_{\lambda}=T\left(\epsilon_{-\lambda}\right)$ for all $\lambda>\omega$. Then, for all $\lambda, \mu \in(\omega, \infty)$,

$$
(\mu-\lambda) T\left(\epsilon_{-\lambda} * \epsilon_{-\mu}\right)=T\left(\epsilon_{-\lambda}-\epsilon_{-\mu}\right)=T\left(\epsilon_{-\lambda}\right)-T\left(\epsilon_{-\mu}\right)
$$


because $\epsilon$ is a pseudo-resolvent, and

$$
(\mu-\lambda) T\left(\epsilon_{-\lambda}\right) T\left(\epsilon_{-\mu}\right)=(\mu-\lambda) r_{\lambda} r_{\mu}=r_{\lambda}-r_{\mu}=T\left(\epsilon_{-\lambda}\right)-T\left(\epsilon_{-\mu}\right)
$$

because $r$ is a pseudo-resolvent. Hence $T\left(\epsilon_{-\lambda} * \epsilon_{-\mu}\right)=T\left(\epsilon_{-\lambda}\right) T\left(\epsilon_{-\mu}\right)$ for all $\lambda, \mu \in$ $(\omega, \infty)$ with $\lambda \neq \mu$. This identity readily extends to the case $\lambda=\mu$, since the mapping $(\omega, \infty) \ni \lambda \mapsto \epsilon_{-\lambda} \in L^{1}\left(\mathbb{R}_{+}, \Omega\right)$ is continuous. Accordingly, $T(f * g)=T(f) T(g)$ for all $f, g \in P\left(\mathbb{R}_{+}, \omega\right)$. Since $P\left(\mathbb{R}_{+}, \omega\right)$ is dense in $L^{1}\left(\mathbb{R}_{+}, \Omega\right)$ and $T$ is bounded, we have $T(f * g)=T(f) T(g)$ for all $f, g \in L^{1}\left(\mathbb{R}_{+}, \mathrm{m}\right)$. Thus $T$ is a homomorphism from $L^{1}\left(\mathbb{R}_{+}, \Omega\right)$ to $\boldsymbol{A}$.

Coupled with Theorem 1.2, these observations lead to the following result.

Theorem 5.1. Let $\boldsymbol{A}$ be a Banach algebra, let $\Omega$ be a weight function on $\mathbb{R}_{+}$with bound $\omega$, and let $r:(\omega, \infty) \rightarrow \boldsymbol{A}, \lambda \mapsto r_{\lambda}$, be a pseudo-resolvent. Then $r$ satisfies the Hille-Yosida condition

$$
\|r\|_{W, \Omega, \omega}=\sup \left\{\left\|r_{\lambda}^{k+1}\right\| /\left\|\alpha_{k, \lambda}\right\|_{1, \Omega} \mid k \in \mathbb{Z}^{+}, \lambda \in(\omega, \infty)\right\}<\infty
$$

if and only if there exists a bounded homomorphism $T: L^{1}\left(\mathbb{R}_{+}, \Omega\right) \rightarrow \boldsymbol{A}$ such that $T\left(\epsilon_{-\lambda}\right)=r_{\lambda}$ for each $\lambda \in(\omega, \infty)$. Furthermore, if there exists a bounded homomorphism $T: L^{1}\left(\mathbb{R}_{+}, \Omega\right) \rightarrow \boldsymbol{A}$ such that $T\left(\epsilon_{-\lambda}\right)=r_{\lambda}$ for each $\lambda \in(\omega, \infty)$, then $T$ is unique and $\|T\|=\|r\|_{W, \Omega, \omega}$.

In the case when $\Omega$ is an $(\alpha, \omega)$-weight function, this theorem is due to Kisyński [17]. Related results can be found in $[\mathbf{3}, \mathbf{5}, \mathbf{1 6}]$.

The significance of Theorem 5.1 is that it can be used to develop a generalization of the Hille-Yosida theorem, and a generalization of the Trotter-Kato theorem on the convergence of sequences of one-parameter semigroups (cf. [23, Chapter 9, $\S 12$, Theorem 1]). Both these generalizations will involve semigroups $\left\{S_{t}\right\}_{t \in \mathbb{R}_{+}}$satisfying a growth condition of the form $\sup _{t \in \mathbb{R}_{+}}(\Omega(t))^{-1}\left\|S_{t}\right\|<+\infty$, where $\Omega$ is a weight function on $\mathbb{R}_{+}$. The interested reader is referred to $[\mathbf{6}, \mathbf{1 6}]$ for a formulation and validation of generalizations of the Hille-Yosida theorem and Trotter-Kato theorem based on the classical form of the Widder-Arendt theorem. In [17] a further generalization of the Hille-Yosida theorem is derived from a special case of Theorem 5.1 in which $\Omega$ is an $(\alpha, \omega)$-weight function. All of these generalizations of the Hille-Yosida theorem and Trotter-Kato theorem can eventually be subsumed by respective generalizations, derivable from Theorem 5.1, involving weight functions with a bound. A detailed exposition of these latter results will be presented elsewhere.

\section{References}

1. W. AREndT, Vector-valued Laplace transforms and Cauchy problems, Israel J. Math. 59 (1987), 327-352.

2. A. Bobrowski, On the Yosida approximation and the Widder-Arendt representation theorem, Studia Math. 124 (1997), 281-290.

3. A. Bobrowski, The Widder-Arendt theorem on inverting of the Laplace transform and its relationships with the theory of semigroups, Meth. Funct. Analysis Topol. 3 (1997), $1-39$. 
4. A. Bobrowski, Inversion of the Laplace transform and generation of Abel summable semigroups, J. Funct. Analysis 186 (2001), 1-24.

5. W. ChOJnAcki, On the equivalence of a theorem of Kisyński and the Hille-Yosida generation theorem, Proc. Am. Math. Soc. 126 (1998), 491-497.

6. W. Chojnacki, Multiplier algebras, Banach bundles, and one-parameter semigroups, Ann. Scuola Norm. Sup. Pisa Cl. Sci. (4) 28 (1999), 287-322.

7. R. DeLaubenfels, Z. Huang, S. WAng, And Y. Wang, Laplace transforms of polynomially bounded vector-valued functions and semigroups of operators, Israel J. Math. 98 (1997), 189-207.

8. J. Diestel And J. J. Uhl, Vector measures, Mathematical Surveys, no. 15 (American Mathematical Society, Providence, RI, 1977).

9. N. Dinculeanu, Vector measures (Pergamon and VEB Deutscher Verlag der Wissenschaften, Oxford and Berlin, 1967).

10. N. Dunford And J. T. Schwartz, Linear operators, Part I, General theory (Interscience, 1958).

11. N. Dunford And J. T. Schwartz, Linear operators, Part II, Spectral theory, self adjoint operators in Hilbert space (Interscience, 1963).

12. W. FELLER, An introduction to probability theory and its applications, vol. 2 (Wiley, 1971).

13. P. R. Halmos, Measure theory (Springer, 1974).

14. B. HENNIG AND F. NeUBRANDER, On representations, inversions, and approximations of Laplace transforms in Banach spaces, Applicable Analysis 49 (1993), 151-170.

15. E. Hille AND R. S. Phillips, Functional analysis and semi-groups (American Mathematical Society, Providence, RI, 1957).

16. J. KISYŃSKI, The Widder spaces, representations of the convolution algebra $L^{1}\left(\mathbb{R}^{+}\right)$, and one parameter semigroups of operators, Preprint no. 588, Institute of Mathematics, Polish Academy of Science, Warsaw, June 1998.

17. J. KISYŃSKI, Around Widder's characterization of the Laplace transform of an element of $L^{\infty}\left(\mathbb{R}^{+}\right)$, Ann. Polon. Math. 74 (2000), 161-200.

18. F. NEUBRANDER, The Laplace-Stieltjes transform in Banach spaces and abstract Cauchy problems, in Evolution Equations, Control Theory, and Biomathematics, Proc. 3rd Int. Workshop-Conf. on Evolution Equations, Control Theory, and Biomathematics, Han-surLesse, Belgium, 20-26 October 1991 (ed. Ph. Clément and G. Lumer), Lecture Notes in Pure and Applied Mathematics, vol. 155, pp. 417-431 (Basel, Marcel Dekker, 1994).

19. R. S. Phillips, An inversion formula for Laplace transforms and semi-groups of linear operators, Ann. Math. 59 (1954), 325-356.

20. S. SAKS AND A. ZYGmund, Analytic functions (Elsevier and PWN (Polish Scientific Publishers), Amsterdam and Warsaw, 1971).

21. D. V. WidDER, The Laplace transform (Princeton University Press, 1946).

22. D. V. WIDDER, An introduction to transform theory (Academic, 1971).

23. K. YosidA, Functional analysis, 5th edn (Springer, 1978). 